\title{
Constructions of Dense Lattices over Number Fields
}

\author{
A. A. ANDRADE ${ }^{1 *}$, A. J. FERRARI ${ }^{2}$, J. C. INTERLANDO ${ }^{3}$ and R. R. ARAUJO 4 \\ Received on May 15, 2019 / Accepted on September 26, 2019
}

\begin{abstract}
In this work, we present constructions of algebraic lattices in Euclidean space with optimal center density in dimensions $2,3,4,5,6,8$ and 12 , which are rotated versions of the lattices $\Lambda_{n}$, for $n=$ $2,3,4,5,6,8$ and $K_{12}$. These algebraic lattices are constructed through canonical homomorphism via $\mathbb{Z}$ modules of the ring of algebraic integers of a number field.
\end{abstract}

Keywords: algebric lattices, number fields, sphere packings.

\section{INTRODUCTION}

Algebraic number theory has recently raised a great interest for its new role in algebraic lattice theory and in code design for many different coding applications. Algebraic lattices have been useful in information theory and the question of finding algebraic lattices over number fields maximum center density. The problem of finding algebraic lattices with maximal minimum product distance has been studied in last years and this has motived special attention of many researchs in considering ideals of certain rings. The search for dense algebraic lattices in general dimensions has been encouraged in the last decades because they can be applied to Information Theory [1]- [4].

The classical sphere packing problem consists to find out how densely a large number of identical spheres can be packed together in the Euclidean space. The packing density, $\Delta(\Lambda)$, of a lattice $\Lambda$ is the proportion of the space $\mathbb{R}^{n}$ covered by the non-overlapping spheres of maximum radius centered at the points of $\Lambda$. The densest possible lattice packings have only be determined in

\footnotetext{
*Corresponding author: Antonio Aparecido de Andrade - E-mail: antonio.andrade@unesp.br

${ }^{1}$ Departamento de Matemática, Instituto de Biociências, Letras e Ciências Exatas (Ibilce), Universidade Estadual Paulista "Júlio de Mesquita Filho" (Unesp), Campus de São José do Rio Preto - SP, Brasil. E-mail: antonio.andrade@unesp.br https://orcid.org/0000-0001-6452-2236

2 Departamento de Matemática, Faculdade de Ciências (FC), Universidade Estadual Paulista "Júlio de Mesquita Filho" (Unesp), Campus de Bauru - SP, Brasil. E-mail: agnaldo.ferrari@unesp.br https://orcid.org/0000-0002-1422-1416

${ }^{3}$ Department of Mathematics \& Statistics, San Diego State University, San Diego, California, USA. E-mail: interlan@sdu.edu https://orcid.org/0000-0003-4928-043X

${ }^{4}$ Instituto Federal de São Paulo, Cubatão - SP, Brasil. E-mail: dearaujorobsonricardo@ gmail.com https://orcid.org/00000002-1357-9926
} 
dimensions 1 to 8 and 24. It is also known that these densest lattice packings are unique (up to equivalences) [5].

This paper is organized as follows. In Section 2, notions and results from algebraic number theory that are used in the work are reviewed. In Section 3, rotated lattices are constructed from number fields in dimensions $2,3,4,5,6,8$ and 12 , which are rotated versions of the lattices $\Lambda_{n}$, for $n=2,3,4,5,6,8$ and $K_{12}$.

\section{BACKGROUND OF NUMBER FIELDS}

Let $\mathbb{K}$ be a number field, i.e., $\mathbb{K}$ is a finite extension of $\mathbb{Q}$. By Primitive Element Theorem, there is an element $\theta \in \mathbb{K}$ such that $\mathbb{K}=\mathbb{Q}(\theta)=\left\{\sum_{i=0}^{n-1} a_{i} \theta^{i} ; a_{i} \in \mathbb{Q}\right\}$, where $\theta$ is a root of a polynomial $p(x) \in \mathbb{Q}[x]$ of minimal degree $n$. A cyclotomic field is a number field such that $\mathbb{K}=\mathbb{Q}(\boldsymbol{\theta})$, where $\theta$ is a primitive $n$-th root of unity. If $\theta_{1}=\theta, \theta_{2}, \ldots, \theta_{n}$ are the $n$ distinct roots of $p(x)$, then threre are exactly $n$ distinct $\mathbb{Q}$-embeddings $\sigma_{i}: \mathbb{K} \rightarrow \mathbb{C}$ such that $\sigma_{i}(\theta)=\theta_{i}$, for all $i=1,2, \ldots, n$. Furhtermore, there are $r_{1}$ real embeddings $\sigma_{1}, \ldots, \sigma_{r_{1}}$ and $2 r_{2}$ complex embeddings $\sigma_{r_{1}+1}, \overline{\sigma_{r_{1}+1}}, \ldots, \sigma_{r_{1}+r_{2}},{\overline{\sigma_{1}+r_{2}}}_{\text {. If }} \mathfrak{\Re}(x)$ and $\mathfrak{I}(x)$ denote, respectively, the real part and the imaginary part of $x$, the canonical embedding $\sigma: \mathbb{K} \longrightarrow \mathbb{R}^{n}$, with $x \in \mathbb{K}$, is defined by

$$
\sigma(x)=\left(\sigma_{1}(x), \ldots, \sigma_{r_{1}}(x), \mathfrak{A}\left(\sigma_{r_{1}+1}(x)\right), \ldots, \mathfrak{I}\left(\sigma_{r_{1}+r_{2}}(x)\right)\right) .
$$

The set $\mathscr{O}_{\mathbb{K}}=\{\alpha: f(\alpha)=0$ for some monic polynomial $f(x) \in \mathbb{Z}[x]\}$ is a ring called ring of algebraic integers of $\mathbb{K}$. The ring $\mathscr{O}_{\mathbb{K}}$ has a basis $\left\{\alpha_{1}, \alpha_{2}, \ldots, \alpha_{n}\right\}$ over $\mathbb{Z}$. In other words, every element $\alpha \in \mathscr{O}_{\mathbb{K}}$ is uniquely written as $\alpha=\sum_{i=1}^{n} a_{i} \alpha_{i}$, where $a_{i} \in \mathbb{Z}$ for all $i=1,2, \ldots, n$, and every nonzero fractional ideal $\mathscr{M}$ of $\mathscr{O}_{\mathbb{K}}$ is a free $\mathbb{Z}$-module of rank $n$ [7].

If $\alpha \in \mathbb{K}$, the value

$$
\operatorname{Tr}_{\mathbb{K}}(\alpha)=\sum_{i=1}^{n} \sigma_{i}(\alpha)
$$

is called trace of $\alpha$ in $\mathbb{K}$. If $\left\{\alpha_{1}, \alpha_{2}, \ldots, \alpha_{n}\right\}$ is an integral basis of $\mathbb{K}$, the discriminant of $\mathbb{K}$ is defined as $D_{\mathbb{K}}=\operatorname{det}\left[\sigma_{j}\left(\alpha_{i}\right)\right]^{2}$ and it is an invariant over change of basis [6].

\section{CONSTRUCTIONS OF DENSE ALGEBRAIC LATTICES}

A lattice $\Lambda$ is a discrete additive subgroup of $\mathbb{R}^{n}$, that is, $\{0\} \neq \Lambda \subseteq \mathbb{R}^{n}$ is a lattice iff there are linearly independent vectors $\left\{v_{1}, v_{2}, \ldots, v_{k}\right\}$, with $k \leq n$, in $\mathbb{R}^{n}$ such that

$$
\Lambda=\left\{\sum_{i=1}^{k} a_{i} v_{i}: a_{i} \in \mathbb{Z}, \text { for all } i=1,2, \ldots, k\right\} .
$$

The set $\left\{v_{1}, v_{2}, \ldots, v_{k}\right\}$ is called a basis for $\Lambda$, the matrix $M$ whose rows are these vectors is called a generator matrix for $\Lambda$ and the matrix $G=M M^{t}$ is called Gram matrix. 
If $\mathscr{M}$ is a $\mathbb{Z}$-submodule in $\mathbb{K}$ of rank $n$, the set $\Lambda=\sigma(\mathscr{M})$ is a lattice in $\mathbb{R}^{n}$ called an algebraic lattice. The center density of $\Lambda$ is given by

$$
\delta(\Lambda)=\frac{t^{n / 2}}{2^{n}\left[\mathscr{O}_{\mathbb{K}}: \mathscr{M}\right] \sqrt{\left|D_{\mathbb{K}}\right|}},
$$

where $t=\min \left\{\operatorname{Tr}_{\mathbb{K}}(\alpha \bar{\alpha}): \alpha \in \mathscr{M}, \alpha \neq 0\right\}$ and $\left[\mathscr{O}_{\mathbb{K}}: \mathscr{M}\right]$ denotes the index of the submodule $\mathscr{M}$.

Example 3.1. If $\mathbb{K}=\mathbb{Q}\left(\zeta_{3}\right)$, where $\zeta_{3}$ is the primitive 3-th root of unity, then $[\mathbb{K}: \mathbb{Q}]=2,\left\{1, \zeta_{3}\right\}$ is a basis of $\mathbb{K}$ and $D_{\mathbb{K}}=-3$. If $\mathscr{M}$ is a submodule of $\mathscr{O}_{\mathbb{K}}$ given by

$$
\mathscr{M}=\left\{a_{0}+a_{1} \zeta_{3}: a_{0}, a_{1} \in \mathbb{Z}\right\},
$$

then $\left[\mathscr{O}_{\mathbb{K}}: \mathscr{M}\right]=1$ and

$$
\operatorname{Tr}_{\mathbb{K}}(\alpha \bar{\alpha})=2\left(a_{0}^{2}-a_{0} a_{1}+a_{1}^{2}\right),
$$

where $\alpha \in \mathscr{M}$. Since $t=\min \left\{\operatorname{Tr}_{\mathbb{K}}(\alpha \bar{\alpha}): \alpha \in \mathscr{M}, \alpha \neq 0\right\}=2$ with $a_{0}=1$ and $a_{1}=0$, it follows that

$$
\delta(\mathscr{M})=\frac{(\sqrt{2} / 2)^{2}}{\sqrt{3}}=\frac{1}{2 \sqrt{3}},
$$

i.e., the center density of $\sigma(\mathscr{M})$ is the same of the lattice $\Lambda_{2}$. Similarly, if $\mathbb{K}=\mathbb{Q}(\sqrt{3})$, then $[\mathbb{K}: \mathbb{Q}]=2,\{1, \sqrt{3}\}$ is a basis of $\mathbb{K}$ and $D_{\mathbb{K}}=12$. If $\mathscr{M}$ is a submodule of $\mathscr{O}_{\mathbb{K}}$ given by $\mathscr{M}=\left\{a_{0}+a_{1} \sqrt{3}: a_{0}-a_{1} \equiv 0(\bmod 2)\right.$ and $\left.a_{0}, a_{1} \in \mathbb{Z}\right\}$, then $\left[\mathscr{O}_{\mathbb{K}}: \mathscr{M}\right]=2$ and $\operatorname{Tr}_{\mathbb{K}}\left(\alpha^{2}\right)=8 a_{0}^{2}+24 a_{0} a_{1}+24 a_{1}^{2}$, where $\alpha \in \mathscr{M}$. Since $t=\min \left\{\operatorname{Tr}_{\mathbb{K}}\left(\alpha^{2}\right): \alpha \in \mathscr{M}, \alpha \neq 0\right\}=8$ with $a_{0}=1$ and $a_{1}=0$, it follows that $\delta(\mathscr{M})=\frac{(\sqrt{8} / 2)^{2}}{2^{3} \sqrt{3}}=\frac{1}{4 \sqrt{3}}$.

Example 3.2. If $\mathbb{K}=\mathbb{Q}(\theta)$, where $\theta=\zeta_{9}+\zeta_{9}^{-1}$ and $\zeta_{9}$ is the primitive 9-th root of unity, then $[\mathbb{K}: \mathbb{Q}]=3,\left\{1, \theta, \theta^{2}\right\}$ is an integral basis of $\mathbb{K}$ and $D_{\mathbb{K}}=3^{4}$. If $\mathscr{M}$ is a submodule of $\mathscr{O}_{\mathbb{K}}$ given by

$$
\begin{aligned}
\mathscr{M}= & \left\{a_{0}+a_{1} \theta+a_{2} \theta^{2}: a_{0} \equiv 0(\bmod 2) \text { and } a_{0}+2 a_{1}+a_{2} \equiv 0(\bmod 3),\right. \text { where } \\
& \left.a_{0}, a_{1}, a_{2} \in \mathbb{Z}\right\},
\end{aligned}
$$

then $\left[\mathscr{O}_{\mathbb{K}}: \mathscr{M}\right]=6$ and

$$
\operatorname{Tr}_{\mathbb{K}}\left(\alpha^{2}\right)=18\left(a_{0}^{2}+a_{0} a_{1}+5 a_{0} a_{2}+a_{1}^{2}+5 a_{1} a_{2}+9 a_{2}^{2}\right),
$$

where $\alpha \in \mathscr{M}$. Since $t=\min \left\{\operatorname{Tr}_{\mathbb{K}}\left(\alpha^{2}\right): \alpha \in \mathscr{M}, \alpha \neq 0\right\}=18$ with $a_{0}=1$ and $a_{1}=a_{2}=0$, it follows that

$$
\delta(\mathscr{M})=\frac{(\sqrt{18} / 2)^{3}}{54}=\frac{1}{4 \sqrt{2}},
$$

i.e., the center density of $\sigma(\mathscr{M})$ is the same of the lattice $\Lambda_{3}$. Similarly, if $\mathscr{M}=\left\{a_{0}+a_{1} \theta+a_{2} \theta^{2}: a_{0} \equiv 0(\bmod 2)\right.$ and $a_{0}+2 a_{1}+a_{2} \equiv 0(\bmod 3)$, where $\left.a_{0}, a_{1}, a_{2} \in \mathbb{Z}\right\}$, then $\left[\mathscr{O}_{\mathbb{K}}: \mathscr{M}\right]=6$ and $\operatorname{Tr}_{\mathbb{K}}\left(\alpha^{2}\right)=18\left(3 a_{0}^{2}+3 a_{0} a_{1}+10 a_{0} a_{2}+a_{1}^{2}+5 a_{1} a_{2}+9 a_{2}^{2}\right)$, 
where $\alpha \in \mathscr{M}$. Since $t=\min \left\{\operatorname{Tr}_{\mathbb{K}}\left(\alpha^{2}\right): \alpha \in \mathscr{M}, \alpha \neq 0\right\}=18$ with $a_{0}=a_{2}=0$ and $a_{1}=1$, it follows that $\delta(\mathscr{M})=\frac{(\sqrt{18} / 2)^{3}}{2 \cdot 3 \cdot 3^{2}}=\frac{1}{4 \sqrt{2}}$. Similarly, if $\mathscr{M}=\left\{a_{0}+a_{1} \theta+a_{2} \theta^{2}\right.$ : $a_{0} \equiv 0(\bmod 2) \quad$ and $a_{0}+2 a_{1}+a_{2} \equiv 0(\bmod 3)$, where $\left.a_{0}, a_{1}, a_{2} \in \mathbb{Z}\right\}$, then $\left[\mathscr{O}_{\mathbb{K}}: \mathscr{M}\right]=6$ and $\operatorname{Tr}_{\mathbb{K}}\left(\alpha^{2}\right)=18\left(3 a_{0}^{2}+3 a_{0} a_{1}+10 a_{0} a_{2}+a_{1}^{2}+5 a_{1} a_{2}+9 a_{2}^{2}\right)$, where $\alpha \in \mathscr{M}$. Since $t=\min \left\{\operatorname{Tr}_{\mathbb{K}}\left(\alpha^{2}\right): \alpha \in \mathscr{M}, \alpha \neq 0\right\}=18$ with $a_{0}=a_{2}=0$ and $a_{1}=1$, it follows that $\delta(\mathscr{M})=\frac{(\sqrt{18} / 2)^{3}}{2 \cdot 3 \cdot 3^{2}}=\frac{1}{4 \sqrt{2}}$. Finally, if $\mathbb{K}=\mathbb{Q}(\theta)$, where $\theta$ is a root of $p(x)=x^{3}-3 x+1$, then $[\mathbb{K}: \mathbb{Q}]=3,\left\{1, \theta, \theta^{2}\right\}$ is a basis of $\mathbb{K}$ and $D_{\mathbb{K}}=3^{4}$. If $\mathscr{M}$ is a submodule of $\mathscr{O}_{\mathbb{K}}$ given by $\mathscr{M}=\left\{a_{0}+a_{1} \theta+a_{2} \theta^{2}: a_{2} \equiv 0(\bmod 2)\right.$ and $a_{0}-a_{1}+a_{2} \equiv 0(\bmod 3)$, with $\left.a_{0}, a_{1}, a_{2} \in \mathbb{Z}\right\}$, then $\left[\mathscr{O}_{\mathbb{K}}: \mathscr{M}\right]=6$ and $\operatorname{Tr}_{\mathbb{K}}\left(\alpha^{2}\right)=18\left(a_{0}^{2}+5 a_{0} a_{1}+3 a_{0} a_{2}+9 a_{1}^{2}+10 a_{1} a_{2}+3 a_{2}^{2}\right)$, where $\alpha \in \mathscr{M}$. Since $t=\min \left\{\operatorname{Tr}_{\mathbb{K}}\left(\alpha^{2}\right): \alpha \in \mathscr{M}, \alpha \neq 0\right\}=18$ with $a_{0}=1$ and $a_{1}=a_{2}=0$, it follows that $\delta(\mathscr{M})=\frac{(\sqrt{18} / 2)^{3}}{2 \cdot 3 \cdot 3^{2}}=\frac{1}{4 \sqrt{2}}$. Similarly, if $\mathbb{K}=\mathbb{Q}(\theta)$, where $\theta=\zeta_{7}+\zeta_{7}^{-1}$ and $\zeta_{7}$ is the primitive 7-th root of unity, then $[\mathbb{K}: \mathbb{Q}]=3,\left\{1, \theta, \theta^{2}\right\}$ is a basis of $\mathbb{K}$ and $D_{\mathbb{K}}=7^{2}$. If $\mathscr{M}$ is a submodule of $\mathscr{O}_{\mathbb{K}}$ given by $\mathscr{M}=\left\{a_{0}+a_{1} \theta+a_{2} \theta^{2}: a_{0} \equiv 0(\bmod 7)\right.$ and $3 a_{1}-a_{2} \equiv 0(\bmod 7)$, with $\left.a_{0}, a_{1}, a_{2} \in \mathbb{Z}\right\}$, then $\left[\mathscr{O}_{\mathbb{K}}: \mathscr{M}\right]=2 \dot{7}^{2}$ and $\operatorname{Tr}_{\mathbb{K}}\left(\alpha^{2}\right)=98\left(a_{0}^{2}-a_{0} a_{2}+a_{1}^{2}+7 a_{1} a_{2}+13 a_{2}^{2}\right)$, where $\alpha \in \mathscr{M}$. Since $t=\min \left\{\operatorname{Tr}_{\mathbb{K}}\left(\alpha^{2}\right): \alpha \in \mathscr{M}, \alpha \neq 0\right\}=98$ with $a_{0}=1$ and $a_{1}=a_{2}=0$, it follows that $\delta(\mathscr{M})=\frac{(\sqrt{98} / 2)^{3}}{2 \cdot 7^{3}}=\frac{1}{4 \sqrt{2}}$.

Example 3.3. If $\mathbb{K}=\mathbb{Q}\left(\zeta_{8}\right)$, where $\zeta_{8}$ is the primitive 8 -th root of unity, then $[\mathbb{K}: \mathbb{Q}]=4,\left\{1, \zeta_{8}, \zeta_{8}^{2}, \zeta_{8}^{3}\right\}$ is an integral basis of $\mathbb{K}$ and $D_{\mathbb{K}}=2^{8}$. If $\mathscr{M}$ is a submodule of $\mathscr{O}_{\mathbb{K}}$ given by

$$
\mathscr{M}=\left\{a_{0}+a_{1} \zeta_{8}+a_{2} \zeta_{8}^{2}+a_{3} \zeta_{8}^{3}: a_{0}+a_{1}+a_{2}+a_{3} \equiv 0(\bmod 2), \text { where } a_{0}, a_{1}, a_{2}, a_{3} \in \mathbb{Z}\right\},
$$

then $\left[\mathscr{O}_{\mathbb{K}}: \mathscr{M}\right]=2$ and

$$
\operatorname{Tr}_{\mathbb{K}}(\alpha \bar{\alpha})=8\left(2 a_{0}^{2}-2 a_{0} a_{3}+a_{1}^{2}-a_{1} a_{2}+a 2^{2}-2 a_{2} a_{3}+2 a_{3}^{2}\right),
$$

where $\alpha \in \mathscr{M}$. Since $t=\min \left\{\operatorname{Tr}_{\mathbb{K}}\left(\alpha^{2}\right): \alpha \in \mathscr{M}, \alpha \neq 0\right\}=8$ with $a_{1}=1$ and $a_{0}=a_{2}=a_{3}=0$, it follows that

$$
\delta(\mathscr{M})=\frac{(\sqrt{8} / 2)^{4}}{32}=\frac{1}{8},
$$

i.e., the center density of $\sigma(\mathscr{M})$ is the same of the lattice $\Lambda_{4}$. Similarly, if $\mathscr{M}=\left\{a_{0}+a_{1} \zeta_{8}+a_{2} \zeta_{8}^{2}+a_{3} \zeta_{8}^{3}: a_{2}+a_{3} \equiv 0(\bmod 2)\right.$, where $\left.a_{0}, a_{1}, a_{2}, a_{3} \in \mathbb{Z}\right\}$, then $\left[\mathscr{O}_{\mathbb{K}}: \mathscr{M}\right]=2$ and $\operatorname{Tr}_{\mathbb{K}}\left(\alpha^{2}\right)=8\left(a_{0}^{2}+a_{1}^{2}+a_{2}^{2}+2 a_{3}^{2}+a_{0} a_{2}+2 a_{0} a_{3}+a_{1} a_{2}+2 a_{2} a_{3}\right)$, where $\alpha \in \mathscr{M}$. Since $t=\min \left\{\operatorname{Tr}_{\mathbb{K}}\left(\alpha^{2}\right): \alpha \in \mathscr{M}, \alpha \neq 0\right\}=8$ with $a_{0}=1$ and $a_{1}=a_{2}=a_{3}=0$, it follows that $\delta(\mathscr{M})=\frac{(\sqrt{8} / 2)^{4}}{32}=\frac{1}{8}$. Similarly, if $\mathbb{K}=\mathbb{Q}(\theta)$, where $\theta$ is a root of $p(x)=x^{4}+3 x^{2}+1$, then $[\mathbb{K}: \mathbb{Q}]=4$, where $\left\{1, \theta, \theta^{2}, \theta^{3}\right\}$ is an integral basis of $\mathbb{K}, D_{\mathbb{K}}=2^{4} \dot{5}^{2}$. If $\mathscr{M}$ is a submodule of $\mathscr{O}_{\mathbb{K}}$ given by $\mathscr{M}=\left\{a_{0}+a_{1} \theta+a_{2} \theta^{2}+a_{3} \theta^{3}: a_{0}-2 a_{1}+2 a_{2}-a_{3} \equiv\right.$ $0(\bmod 5)$, where $\left.a_{0}, a_{1}, a_{2}, a_{3} \in \mathbb{Z}\right\}$, then $\left[\mathscr{O}_{\mathbb{K}}: \mathscr{M}\right]=10$, and if $\alpha \in \mathscr{M}$, then $\operatorname{Tr}_{\mathbb{K}}(\alpha \bar{\alpha})=$ $40 a_{0}^{2}-40 a_{0} a_{1}+132 a_{0} a_{2}+360 a_{0} a_{3}+20 a_{1}^{2}-28 a_{1} a_{2}-140 a_{1} a_{3}+158 a_{2}^{2}+720 a_{2} a_{3}+900 a_{3}^{2}$. Since $t=\min \left\{\operatorname{Tr}_{\mathbb{K}}\left(\alpha^{2}\right): \alpha \in \mathscr{M}, \alpha \neq 0\right\}=20$ with $a_{0}=a_{2}=a_{3}=0$ and $a_{1}=1$, iffollows that $\delta(\mathscr{M})=\frac{(\sqrt{20} / 2)^{4}}{2^{3} \cdot 5^{2}}=\frac{1}{8}$. 
Example 3.4. If $\mathbb{K}=\mathbb{Q}(\theta)$, where $\theta=\zeta_{44}^{10}-\zeta_{44}^{12}$ and $\zeta_{44}$ is the primitive 44-th root of unity, then $[\mathbb{K}: \mathbb{Q}]=5,\left\{1, \theta, \theta^{2}, \theta^{3}, \theta^{4}\right\}$ is an integral basis of $\mathbb{K}$ and the discriminant of $\mathbb{K}$ is $11^{4}$. Let $\mathscr{M}$ be a submodule of $\mathscr{O}_{\mathbb{K}}$ given by

$$
\begin{aligned}
\mathscr{M}= & \left\{a_{0}+a_{1} \theta+a_{2} \theta^{2}+a_{3} \theta^{3}+a_{4} \theta^{4}: a_{0} \equiv 0(\bmod 11), 5 a_{2}+a_{3} \equiv 0(\bmod 11)\right. \\
& \text { and } \left.a_{0}+15 a_{1}+11 a_{2}+a_{4} \equiv 0(\bmod 22), \text { where } a_{0}, a_{1}, a_{2}, a_{3}, a_{4} \in \mathbb{Z}\right\} .
\end{aligned}
$$

In this case, $\mathscr{M}$ is a submodule of $\mathscr{O}_{\mathbb{K}}$ of index $2 \cdot 11^{3}$ and the trace form of $\alpha \in \mathscr{M}$ is given by

$$
\begin{aligned}
\operatorname{Tr}_{\mathbb{K} / \mathbb{Q}}\left(\alpha^{2}\right)= & 37752 a_{0}^{2}+43802 a_{0} a_{1}+79134 a_{0} a_{2}+16456 a_{0} a_{3}+136488 a_{0} a_{4} \\
& +12826 a_{1}^{2}+46706 a_{1} a_{2}+10406 a_{1} a_{3}+79860 a_{1} a_{4}+44286 a_{2}^{2} \\
& +26136 a_{2} a_{3}+144716 a_{2} a_{4}+9438 a_{3}^{2}+30976 a_{3} a_{4}+124388 a_{4}^{2}
\end{aligned}
$$

Thus, $t=\min \left\{\operatorname{Tr}_{\mathbb{K}}\left(\alpha^{2}\right): \alpha \in \mathscr{M}, \alpha \neq 0\right\}=242$ with $a_{0}=a_{2}=a_{3}=0, a_{1}=-3$ and $a_{4}=1$. As the volume of the lattice $\sigma($ mathcalM $)$ is $\sqrt{\left|D_{\mathbb{K}}\right|}\left[\mathscr{M}: \mathscr{O}_{\mathbb{K}}\right]=2 \cdot 11^{5}$, it follows that

$$
\delta(\mathscr{M})=\frac{(\sqrt{242} / 2)^{5}}{2 \cdot 11^{5}}=\frac{1}{8 \sqrt{2}}
$$

i.e., the center density of $\sigma(\mathscr{M})$ is the same of the lattice $\Lambda_{7}$.

Example 3.5. If $\mathbb{K}=\mathbb{Q}\left(\zeta_{9}\right)$, where $\zeta_{9}$ is the primitive 9-th root of unity, then $[\mathbb{K}: \mathbb{Q}]=6,\left\{1, \zeta_{9}, \zeta_{9}^{2}, \zeta_{9}^{3}, \zeta_{9}^{4}, \zeta_{9}^{5}\right\}$ is an integral basis of $\mathbb{K}$ and $D_{\mathbb{K}}=-3^{9}$. If $\mathscr{M}$ is a submodule of $\mathscr{O}_{\mathbb{K}}$ given by

$$
\begin{aligned}
\mathscr{M}= & \left\{a_{0}+a_{1} \zeta_{9}+a_{2} \zeta_{9}^{2}+a_{3} \zeta_{9}^{3}+a_{4} \zeta_{9}^{4}+a_{5} \zeta_{9}^{5}: a_{1}-a_{2}+a_{4}-a_{5} \equiv 0(\bmod 3),\right. \\
& \text { where } \left.a_{0}, a_{1}, \ldots, a_{5} \in \mathbb{Z}\right\},
\end{aligned}
$$

then $\left[\mathscr{O}_{\mathbb{K}}: \mathscr{M}\right]=9$ and

$$
\begin{aligned}
\operatorname{Tr}_{\mathbb{K}}(\alpha \bar{\alpha})= & 18\left(a_{0}^{2}+a_{0} a_{1}+a_{0} a_{2}+a_{0} a_{3}+2 a_{0} a_{4}+2 a_{0} a_{5}+a_{1}^{2}+a_{1} a_{3}+3 a_{1} a_{4}\right. \\
& +a_{2}^{2}+a_{2} a_{3}+3 a_{2} a_{5}+a_{3}^{2}+2 a_{3} a_{4}+2 a_{3} a_{5}+3 a_{4}^{2}+3 a_{5}^{2}
\end{aligned}
$$

where $\alpha \in \mathscr{M}$. Since $t=\min \left\{\operatorname{Tr}_{\mathbb{K}}(\alpha \bar{\alpha}): \alpha \in \mathscr{M}, \alpha \neq 0\right\}=18$ with $a_{0}=1$ and $a_{1}=a_{2}=a_{3}=$ $a_{4}=a_{5}=0$, it follows that

$$
\delta(\mathscr{M})=\frac{(\sqrt{18} / 2)^{6}}{3^{6} \sqrt{3}}=\frac{1}{8 \sqrt{3}},
$$

i.e., the center density of $\sigma(\mathscr{M})$ is the same of the lattice $\Lambda_{6}$.

Example 3.6. If $\mathbb{K}=\mathbb{Q}\left(\zeta_{20}\right)$, where $\zeta_{20}$ is the primitive 20-th root of unity then $[\mathbb{K}: \mathbb{Q}]=8, \quad\left\{1, \zeta_{20}, \zeta_{20}^{2}, \zeta_{20}^{3}, \zeta_{20}^{4}, \zeta_{20}^{5}, \zeta_{20}^{6}, \zeta_{20}^{7}\right\}$ is an integral basis fo $\mathbb{K}$ and $D_{\mathbb{K}}=2^{8} \cdot 5^{6}$. If $\mathscr{M}$ is a submodule of $\mathscr{O}_{\mathbb{K}}$ given by

$$
\begin{aligned}
\mathscr{M}= & \left\{a_{0}+a_{1} \zeta_{20}+a_{2} \zeta_{20}^{2}+a_{3} \zeta_{20}^{3}+a_{4} \zeta_{20}^{4}+a_{5} \zeta_{20}^{5}+a_{6} \zeta_{20}^{6}+a_{7} \zeta_{20}^{7}: a_{0}+a_{4} \equiv 0(\bmod 4)\right. \\
& a_{1}+a_{5} \equiv 0(\bmod 2), a_{2}+a_{3}+a_{6} \equiv 0(\bmod 4) \text { and } a_{7} \equiv 0(\bmod 5), \text { where } \\
& \left.a_{0}, a_{1}, \ldots, a_{7} \in \mathbb{Z}\right\}
\end{aligned}
$$


then $\left[\mathscr{O}_{\mathbb{K}}: \mathscr{M}\right]=5$ and

$$
\begin{aligned}
\operatorname{Tr}_{\mathbb{K}}(\alpha \bar{\alpha})= & 20\left(2 a_{0}^{2}+2 a_{0} a_{1}+5 a_{0} a_{2}+3 a_{0} a_{3}+3 a_{0} a_{4}+2 a_{0} a_{5}+5 a_{0} a_{6}+8 a_{0} a_{7}+a_{1}^{2}\right. \\
& +3 a_{1} a_{2}+2 a_{1} a_{3}+2 a_{1} a_{4}+a_{1} a_{5}+3 a_{1} a_{6}+5 a_{1} a_{7}+4 a 2^{2}+4 a_{2} a_{3}+5 a_{2} a_{4} \\
& +3 a_{2} a_{5}+7 a_{2} a_{6}+12 a_{2} a_{7}+2 a_{3}^{2}+3 a_{3} a_{4}+2 a_{3} a_{5}+5 a_{3} a_{6}+7 a_{3} a_{7}+2 a_{4}^{2} \\
& \left.+2 a_{4} a_{5}+5 a_{4} a_{6}+8 a_{4} a_{7}+a_{5}^{2}+3 a_{5} a_{6}+5 a_{5} a_{7}+4 a_{6}^{2}+12 a_{6} a_{7}+10 a_{7}^{2}\right),
\end{aligned}
$$

where $\alpha \in \mathscr{M}$. Since $t=\min \left\{\operatorname{Tr}_{\mathbb{K}}(\alpha \bar{\alpha}): \alpha \in \mathscr{M}, \alpha \neq 0\right\}=20$ with $a_{1}=1$ and $a_{0}=a_{2}=a_{3}=$ $a_{4}=a_{5}=a_{6}=a_{7}=0$, it follows that

$$
\delta(\mathscr{M})=\frac{(\sqrt{20} / 2)^{8}}{2^{4} \cdot 5^{4}}=\frac{1}{16},
$$

i.e., the center density of $\sigma(\mathscr{M})$ is the same of the lattice $\Lambda_{8}$.

Example 3.7. If $\mathbb{K}=\mathbb{Q}\left(\zeta_{21}\right)$, where $\zeta_{21}$ is the primitive 21 -th root of unity, then $[\mathbb{K}: \mathbb{Q}]=12$, $\left\{1, \zeta_{21}, \ldots, \zeta_{21}^{11}\right\}$ is an integral basis of $\mathbb{K}$ and $D_{\mathbb{K}}=3^{6} \cdot 7^{10}$. If $\mathscr{M}$ is a submodule of $\mathscr{O}_{\mathbb{K}}$ given by

$$
\begin{aligned}
\mathscr{M}= & \left(\zeta_{21}^{6}-\zeta_{21}^{2}+1\right) a_{0}+\left(\zeta_{21}^{7}-\zeta_{21}^{3}+\zeta_{21}\right) a_{1}+\left(\zeta_{21}^{8}-\zeta_{21}^{4}+\zeta_{21}^{2}\right) a_{2} \\
& +\left(\zeta_{21}^{9}-\zeta_{21}^{5}+\zeta_{21}^{3}\right) a_{3}+\left(\zeta_{21}^{10}-\zeta_{21}^{6}+\zeta_{21}^{4}\right) a_{4}+\left(\zeta_{21}^{1} 1-\zeta_{21}^{7}+\zeta_{21}^{5}\right) a_{5} \\
& +\left(\zeta_{21}^{11}-\zeta_{21}^{9}+\zeta_{21}^{4}-\zeta_{21}^{3}+\zeta_{21}-1\right) a_{6} \\
& +\left(\zeta_{21}^{11}-\zeta_{21}^{10}-\zeta_{21}^{9}+\zeta_{21}^{8}-\zeta_{21}^{6}+\zeta_{21}^{5}-\zeta_{21}^{3}+\zeta_{21}^{2}-1\right) a_{7} \\
& +\left(-\zeta_{21}^{10}+\zeta_{21}^{8}-\zeta_{21}^{7}-1\right) a_{8}+\left(-\zeta_{21}^{11}+\zeta_{21}^{9}-\zeta_{21}^{8}-\zeta_{21}\right) a_{9} \\
& +\left(-\zeta_{21}^{11}+\zeta_{21}^{10}-\zeta_{21}^{8}+\zeta_{21}^{6}-\zeta_{21}^{4}+\zeta_{21}^{3}-\zeta_{21}^{2}-\zeta_{21}+1\right) a_{10} \\
& \left.+\left(-\zeta_{21}^{8}+\zeta_{21}^{7}+\zeta_{21}^{6}-\zeta_{21}^{5}-\zeta_{21}^{2}+1\right) a_{11}, \text { where } a_{0}, a_{1}, \ldots, a_{11} \in \mathbb{Z}\right\},
\end{aligned}
$$

then $\left[\mathscr{O}_{\mathbb{K}}: \mathscr{M}\right]=7$ and

$$
\begin{aligned}
\operatorname{Tr}_{\mathbb{K}}(\alpha \bar{\alpha})= & 28 a_{0}^{2}-14 a_{0} a_{2}-14 a_{0} a_{3}-14 a_{0} a_{4}+28 a_{0} a_{5}-28 a_{0} a_{7}-14 a_{0} a_{9} \\
& +28 a_{0} a_{10}+28 a_{0} a_{11}+28 a_{1}^{2}-14 a_{1} a_{3}-14 a_{1} a_{4}-14 a_{1} a_{5}+28 a_{1} a_{6} \\
& -28 a_{1} a_{8}-14 a_{1} a_{10}+28 a_{1} a_{11}+28 a_{2}^{2}-14 a_{2} a_{4}-14 a_{2} a_{5}-14 a_{2} a_{6} \\
& +28 a_{2} a_{7}-28 a_{2} a_{9}-14 a_{2} a_{11}+28 a_{3}^{2}-14 a_{3} a_{5}-14 a_{3} a_{6}-14 a_{3} a_{7} \\
& +28 a_{3} a_{8}-28 a_{3} a_{10}+28 a_{4}^{2}-14 a_{4} a_{6}-14 a_{4} a_{7}-14 a_{4} a_{8}+28 a_{4} a_{9} \\
& -28 a_{4} a_{11}+28 a_{5}^{2}-14 a_{5} a_{7}-14 a_{5} a_{8}-14 a_{5} a_{9}+28 a_{5} a_{10}+28 a_{6}^{2} \\
& -14 a_{6} a_{8}-14 a_{6} a_{9}-14 a_{6} a_{10}+28 a_{6} a_{11}+28 a_{7}^{2}-14 a_{7} a_{9}-14 a_{7} a_{10} \\
& -14 a_{7} a_{11}+28 a_{8}^{2}-14 a_{8} a_{10}-14 a_{8} a_{11}+28 a_{9}^{2}-14 a_{9} a_{11}+28 a_{10}^{2} \\
& +28 a_{11}^{2},
\end{aligned}
$$

where $\alpha \in \mathscr{M}$. Since $t=\min \left\{\operatorname{Tr}_{\mathbb{K}}(\alpha \bar{\alpha}): \alpha \in \mathscr{M}, \alpha \neq 0\right\}=28$ with $a_{0}=1$ and $a_{1}=a_{2}=\cdots=$ $a_{11}=0$, it follows that

$$
\delta(\mathscr{M})=\frac{(\sqrt{28} / 2)^{12}}{3^{3} \cdot 5^{6}}=\frac{1}{3^{3}},
$$

i.e., the center density of $\sigma(\mathscr{M})$ is the same of the lattice $K_{12}$. 


\section{ACKNOWLEDGMENT}

The authors thank the reviewer for carefully reading the manuscript and for all the suggestions that improved the presentation of this work. The authors also thank FAPESP 2013/25977-7 and CNPq 429346/2018-2 for its financial support.

RESUMO. Neste trabalho, apresentamos construções de reticulados algébricos no espaço euclidiano com densidade central ótima nas dimensões $2,3,4,5,6,8$ e 12, que são versões rotacionadas dos reticulados $\Lambda_{n}$, para $n=2,3,4,5,6,8$ e $K_{12}$, onde esses reticulados algébricos são construídos através do homomorfismo canônico via $\mathbb{Z}$-módulos do anel de inteiros algébricos de um corpo de números.

Palavras-chave: reticulados algébricos, corpos de números, empacotamento esférico.

\section{REFERENCES}

[1] A.A. Andrade \& R. Palazzo Jr. Linear codes over finite rings. TEMA - Trends in Applied and Computational Mathematics, 6(2) (2005), 207-217.

[2] A.S. Ansari, R. Shah, Zia Ur-Rahman \& A.A. Andrade. Sequences of primitive and nonprimitive BCH codes. TEMA - Trends in Applied and Computational Mathematics, 19(2) (2018), 369-389.

[3] A. A. Andrade, A. J. Ferrari \& C. W. O. Benedito, Constructions of algebraic lattices, Comput. Appl. Math., 29 (2010) 1-13.

[4] E. Bayer-Fluckiger, Lattices and number fields, In: Contemp. Math., Amer. Math. Soc., Providence (1999), 69-84.

[5] J. H. Conway \& N. J. A. Sloane. Sphere Packings, Lattices and Groups, 3rd Edition, Springer Verlag, New York (1999).

[6] J. C. Interlando, J. O. D. Lopes \& T. P .N. Neto. The discriminant of abelian number fields, J. Algebra Appl., 5 (2006), 35-41.

[7] P. Samuel. Algebraic Theory of Numbers, Hermann, Paris (1970). 